\title{
The Innovation Architectures of Facebook
}

\author{
Susan Shepherd Ferebee and James Davis \\ University of Phoenix, Tucson, AZ 85742 \\ United States of America
}

\begin{abstract}
User innovation is enabled through the existence of networks. This article examines the architectures of the social network, Facebook, and provides a theoretical discussion of why and how the architectures of Facebook can support a user innovation environment and what factors of the architectures can improve and enhance innovation.
\end{abstract}

Keywords: innovation, user innovation, social networks, open source.

\section{Introduction}

User innovation is enabled through the existence of networks. Wellman, Boase, and Chen [4] define user communities for participants as "...networks of interpersonal ties that provide sociability, support, information, a sense of belonging, and social identity." It is the mechanism of this connectedness, operating through sociability, mutual support, and exchange of information that underlies the architecture of an important network medium: Facebook. It is our contention that the elements of an effective user innovation process are in place within Facebook. For example, in the presidential election and after he was elected, Barack Obama, his team, and his followers used the design of Facebook and other social networks to innovate in a number of ways: His followers raised half a billion dollars online for the campaign by their grass root movement and communication through the Facebook network. Obama created his own social network, mybarackobama.com, with over 2,000,000 members and continues to use it as a new and innovative communication channel to maintain connection with those who elected him. Obama's team created and maintains Obama profiles in over 15 social network communities Vargas [2] quotes Julius Genachowski, Obama's Chief Technology Advisor, as saying, ""The technology now has made it a lot easier for everyday people to participate." This concept is at the heart of user innovation networks. In addition to the benefit perceived by everyday people in being able to participate, the economic actors, in this case the campaign managers and candidate Obama, derived clear and defined benefits from these innovations. Economic actors, according to von Hippel [3] are defined in terms of the way that actors expect to derive benefit from a given innovation.

\subsection{User Innovation Networks}

According to von Hippel [3], a user innovation network distributes "horizontally" across many product users. Open source software development is an example of a 
user innovation network. Innovation starts with users, not with a manufacturer, and users also contribute to the continuing evolution of the product. Users innovate for their own use but freely share the innovation and all of the details of the design so that others can contribute to the innovation to meet their personal needs, which in turn contributes to the original innovation. The derived benefit to these innovators is not economic, but rather it is creation that meets a personal need (this does not rule out some eventual material gain)

\subsection{The Innovation Architectures of Facebook}

Horizontal distribution is precisely the mechanism that powers Facebook. User innovation architecture is one that allows and encourages exchange and reactivity. The dual architectures of Facebook and its member community represent a framework that enhances knowledge sharing and idea generation. Some examples of the Facebook system architecture are Facebook groups and Facebook applications. Each supports innovation processes through providing unique communication channels that persuade interaction and sharing. Facebook groups can provide a closed group or open group community of shared interests and is an ideal environment for formation of a user innovation network. Applications can be quickly developed as prototypes on Facebook and immediately released for Facebook member use. Feedback and input is rapid, improvements and changes occur quickly, and continuous user involvement in the innovation process is a natural function of the Facebook architecture. Some examples of the Facebook community architecture that support innovation are the values, needs, and attitudes that the members bring to the interactive process.

What is important about the Facebook system frameworks is the open source nature that allows rapid communication, rapid change, and rapid development. The architecture of the Facebook member community, might be described as an architecture of members seeking clarity (how may I input into the system), seeking accuracy (what are the methods of changing the system), and seeking precision (what are the outcomes and benefits to me if I change the system). The Facebook norms, standards, rules, and values evolve when the two persuasive architectures interact.

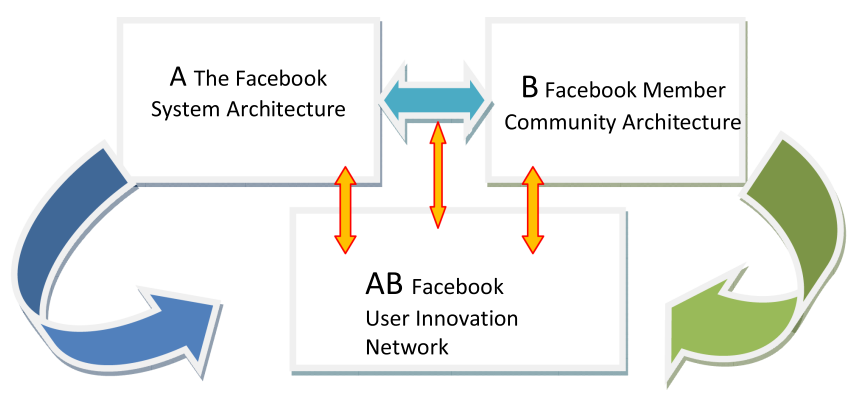

Fig. 1. The Facebook Architecture x Member Community Architecture = Facebook User Innovation Network 
The combined Facebook architectures create the conditions within which innovative behavior will occur. When a member joins a Facebook user innovation group, the two architectures come into proximity. The two architectures influence each other in a series of reactive effects forming an environment where continued contributions begin to equal the return of benefits (reputation, access to knowledge, improved product, possible material returns) for a user innovation network.

\subsection{Facebook Interaction Points}

The two architectures work together through unique interaction points available in the Facebook structure such as the wall, groups, feeds, causes, and applications. These communication channels allow members to quickly share ideas that are available immediately to everyone in the user innovation network or to the whole community, and equally important, allows channels for immediate feedback. Additionally, new status posts and new feeds are automatically broadcast to all members of the innovation network. This factor of automation (a component of the Facebook system architecture) interacts with the community architecture of a user innovation network that is seeking clarity and accuracy through timely information.

\subsection{Reactivity in Facebook}

Reactivity is a response to a stimulus. What is unique about reactivity in social networks, however, is the speed with which the response can occur and the large reach of the response [1]. This is demonstrated by Facebook's recent attempt to change their Terms of Service to state that all content posted by the Facebook community members would be owned by Facebook. In response to this stimulus, immediately, a Facebook Cause group was formed which pushed back against the new Terms of Service. Within days more than 140,000 members had joined the cause. Shortly thereafter, in response to the Facebook community, Facebook relented and reinstated the old Terms of Service.

Reactivity plays a critical role in the success of user innovation groups using a social network like Facebook. As a stimulus (new idea, revised idea) is broadcast to members through automated processes in the Facebook architecture, the stimulus is received simultaneously by a large number of individuals who might then respond with the same speed and broad reach, initiating even more responses. This rapid cycle [1] response leads to an acceleration of innovation.

\subsection{Conclusion}

The reactivity between Facebook and the Facebook community is essential to create the conditions under which innovation can flourish. If the architecture of Facebook is not embraced by the Facebook user innovation community, the innovation possibilities are diminished. If the architecture of the Facebook community is not identified and responded to by Facebook, innovation opportunities would be lost, and the maximization of innovation possibilities will be diminished within the community. The two architectures live or die by their ability to grow together into a Facebook group where sharing and exchange occur. 
When users, the architects of the Facebook community, are given the tools to not only build the new Facebook, but innovate with each other to solve problems, Facebook as a whole becomes a user innovation network.

\section{References}

1. Fogg, B.J.: Mass Interpersonal Persuasion: An Early View of a New Phenomenon. In: Oinas-Kukkonen, H., Hasle, P., Harjumaa, M., Segerståhl, K., Øhrstrøm, P. (eds.) PERSUASIVE 2008. LNCS, vol. 5033, pp. 23-34. Springer, Heidelberg (2008)

2. Vargas, J.: Obama Raised Half A Billion Dollars. The Washington Post (2008), http: //voices.washingtonpost.com/44/2008/11/20/ obama_raised_half_a_billion_on.html

3. VonHippel, E.: Influence, Science and Practice. Infrastructure. Allyn \& Bacon, Boston

4. Wellman, B., Boase, J., Chen, W.: The Networked Nature of Community On and Off the Internet, Working Paper. Center for Urban \& Community Studies. University of Toronto (May 2002) 